Result. This study included 1369 students of which 53\% suffered from PTSD and 62\% from problematic anger. Around 46\% declared a fair or worse general health and $61 \%$ had moderate or severe mental health. Only 9.3\% did not report exposure to any war-related variable. War exposure had an impact on PTSD, anger, and HRQL, but not on students' grades. Smoking, having consanguineous parents, and working did not have a clear association with grades or anger. Social support weakly reduced PTSD and anger scores. Interestingly, working was associatedwith lower PTSD scores but was associated with a worse physical component of HRQL.

Conclusion. This is the largest study on school students in Syria that reports the psychological ramifications of war. Although the direct effects of war could not be precisely described, the high burden of PTSD and anger distress was a strong reflection of the chronic mental distress.

\section{Mental disorder and PTSD in Syria during wartime: a nationwide crisis \\ Sami Jomaa ${ }^{1 \star}$, Ameer Kakaje ${ }^{1}$, Ragheed Al Zohbi², Osama Hosam Aldeen ${ }^{1}$, Leen Makki ${ }^{3}$, Ayham Alyousbashi ${ }^{1}$ and Mhd Bahaa Aldin Alhaffar ${ }^{4}$ \\ ${ }^{1}$ Faculty of Medicine, Damascus University; ${ }^{2}$ Faculty of Medicine, Aleppo University; ${ }^{3}$ Faculty of Medicine, Aleppo University, Department of Experimental Surgery, McGill University and ${ }^{4}$ Department of Periodontology, Faculty of Dentistry, Damascus University \\ ${ }^{\star}$ Corresponding author.}

doi: 10.1192/bjo.2021.696

Aims. Syria has experienced war since 2011, leaving over $80 \%$ under the poverty line and millions displaced. War and its retaliations have significantly impacted the mental health of Syrians. This study evaluates the post-traumatic stress disorder (PTSD), and the severity of the mental distress caused by war and other factors such as low social support. This study also evaluates other variables and compares the findings with those of multiple studies on Syria and refugees.

Method. This is a cross-sectional study that included people who lived in Syria in different governorates. Online surveys were distributed into multiple online groups and included the Kessler 10 (K10) scale which screens for anxiety and depression, the Screen for Posttraumatic Stress Symptoms (SPTSS) tool, the Multidimensional Scale of Perceived Social Support, and questionnaires on demographic and war-related factors.

Result. Our study included 1951 participants, of which, 527 (27.0\%) were males and $1538(78.8 \%)$ between the age of 19 and 25 . Among participants, $44 \%$ had likely severe mental disorder, $27 \%$ had both likely severe mental disorder and full PTSD symptoms, $36.9 \%$ had full PTSD symptoms, and only $10.8 \%$ had neither positive PTSD symptoms nor mental disorder on the K10 scale. Around $23 \%$ had low overall support. Half of the responders were internally displaced, and $27.6 \%$ were forced to change places of living three times or more due to war. Around $86.6 \%$ of the responders believed that the war was the main reason for their mental distress. Those with high SPTSS and K10 scores were found to take more days off from work or school due to negative feelings and having somatic symptoms. Moreover, the number of times changing places of living due to war, educational level, and being distressed by war noise were the most prominent factors for more severe PTSD and mental distress. No differences in PTSD and mental disorder prevalence were noted in participants living in different governorates or among different types of jobs. A strong significant correlation ( $\mathrm{r}$ $=0.623$ ) was found between SPTSS and K10 scores.

Conclusion. The conflict in Syria has left the population at great risk for mental distress which was higher compared to Syrian refugees elsewhere. Many measures with an emphasis on mental health are needed to help the people against a long-term avoidable suffering.

\section{Placebo response in treatment resistant depression:} a systematic review and meta-analysis of multiple treatment modalities

Brett D M Jones ${ }^{1 *}$, Cory R. Weissman ${ }^{1}$, Jewel Karbi ${ }^{1}$, Tya Vine ${ }^{2}$, Louise S. Mulsant ${ }^{3}$, Benoit Mulsant ${ }^{4}$, Andre Brunoni ${ }^{5}$, M. Ishrat Husain ${ }^{6}$, Lais B. Razza ${ }^{5}$, Daniel Blumberger ${ }^{4}$ and Zafiris J Daskalakis ${ }^{7}$

${ }^{1}$ University of Toronto, Department of Psychiatry; ${ }^{2}$ McMaster University; ${ }^{3}$ Nutrition and Food, Nova Scotia Health Authority; ${ }^{4}$ University of Toronto, Department of Psychiatry, Centre for Addiction and Mental Health; ${ }^{5}$ Department of Internal Medicine, Faculty of Medicine, University of Sao Paulo, Laboratory of Neurosciences (LIM-27), Instituto Nacional de Biomarcadores em Neuropsiquiatria (INBioN), Department and Institute of Psychiatry, Faculdade de Medicina, Universit; ${ }^{6}$ Centre for Addiction and Mental Health, University of Toronto, Department of Psychiatry and ${ }^{7}$ Department of Psychiatry, University of California San Diego ${ }^{*}$ Corresponding author.

doi: 10.1192/bjo.2021.697

Aims. The placebo response in depression clinical trials is a major contributing factor for failure to establish the efficacy of novel and repurposed treatments. However, it is not clear as to what the placebo response in treatment-resistant depression (TRD) patients is or whether it differs across treatment modalities. Our objective was to conduct a systematic review and meta-analysis of the magnitude of the placebo response in TRD patients across different treatment modalities and its possible moderators.

Method. Searches were conducted on MEDLINE and PsychInfo from inception to January 24, 2020. Only studies that recruited TRD patients and randomization to a placebo (or sham) arm in a pharmacotherapy, brain stimulation, or psychotherapy study were included (PROSPERO 2020 CRD42020190465). The primary outcome was the Hedges' $g$ for the reported depression scale using a random-effects model. Secondary outcomes included moderators assessed via meta-regression and response and remission rate. Heterogeneity was evaluated using the Egger's Test and a funnel plot. Cochrane Risk of Bias Tool was used to estimate risks.

Result. 46 studies met our inclusion criteria involving a total of 3083 participants (mean (SD) age: 45.7 (6.2); female: 52.4\%). The pooled placebo effect for all modalities was large $(\mathrm{N}=3083$, $\left.\mathrm{g}=1.08,95 \% \mathrm{CI}[0.95-1.20) \mathrm{I}^{2}=0.1\right)$. The placebo effect in studies of specific treatment modalities did not significantly differ: oral medications $\mathrm{g}=1.14$ (95\%CI:0.99-1.29); parenteral medications $\mathrm{g}=1.32$ (95\%CI:0.59-2.04); ayahuasca $\mathrm{g}=0.47$ (95\%CI:-0.281.17); rTMS $\mathrm{g}=0.93$ (95\%CI:0.63-1.23); tDCS $\mathrm{g}=1.32 \quad(95 \%$ CI:0.52-2.11); invasive brain stimulation $\mathrm{g}=1.06$ (95\%CI:0.641.47). There were no psychotherapy trials that met our eligibility criteria. Similarly, response and remission rates were comparable across modalities. Heterogeneity was large. Two variables predicted a lager placebo effect: open-label prospective design (B:0.32, 95\%CI: 0.05-0.58; p:0.02) and sponsoring by a pharmaceutical or medical device company (B:0.39, 95\%CI:0.13-0.65, $\mathrm{p}: 0.004)$ ). No risk of publication bias was found. 
Conclusion. The overall placebo effect in TRD studies was large $(g=1.08)$ and did not differ among treatment modalities. A better understanding of the placebo response in TRD will require: standardizing the definition of TRD, head-to-head comparisons of treatment modalities, an assessment of patient expectations and experiences, and standardized reporting of outcomes.

\section{Mortality rates are higher in lewy body and}

parkinson's disease dementia compared to Alzheimer's dementia in patients referred into a secondary care mental health service. Why?

\author{
Anne $\mathrm{K}^{1 *}$, John O’Brien ${ }^{2}$, Annabel Price ${ }^{1}$, Rudolph Cardinal ${ }^{1}$ \\ and Sinead Moylett ${ }^{2}$ \\ ${ }^{1}$ University of Cambridge, Cambridgeshire and Peterborough NHS \\ foundation trust and ${ }^{2}$ University of Cambridge \\ ${ }^{\star}$ Corresponding author.
}

\section{doi: 10.1192/bjo.2021.698}

Aims. We compared survival in four cohorts of dementia patients- Lewy body (LBD), Parkinson's (PDD), Vascular (VD) and Alzheimer's dementia (AD) - in patients referred into Cambridge and Peterborough NHS Foundation Trust (CPFT) mental health services.

Additionally, we investigated reasons for variation in survival in the four cohorts.

Method. Using electronic records we identified retrospective cohorts of patients referred into services from 2013 onwards. Cases of LBD and PDD were identified using text searches, and comparison cohorts with $\mathrm{AD}$ or VD identified using ICD10 diagnosis codes $\left(\left(\mathrm{F} 00 .^{*}\right)\right.$ or $\left(\mathrm{F} 01 .^{*}\right)$ respectively).

We collected referral (date of referral and service referred into), demographic (date of birth and gender) and diagnosis data on the patients in the four cohorts. Dates of death were available, through central NHS reporting to Trusts.

We used date of first referral as start of the follow-up and end of follow-up, death or 31/12/19. We used Kaplan-Meier and Cox survival analysis to compare survival in the four cohorts.

The cohorts were crossed with Hospital Episode Statistics (HES) data to extract hospital admission diagnoses. We extracted diagnoses of pneumonia due to aspiration and recurrent falls from hospital admissions data using ICD codes (J69.0 and R29.6 respectively). We calculated prevalence of these diagnoses in the dementia groups, in males and females separately.

Result. In Cox analysis (controlling for age at referral, gender and service referred into), the hazard ratio (HR) for death was highest for the PDD group (HR 2.0 (95\% CI 1.7-2.4)), followed by LBD (HR 1.4 (95\% CI 1.3-1.6)), then VD (HR 1.2 (95\% CI 1.0-1.3)), with the $\mathrm{AD}$ group as reference. In the same analysis repeated separately for males and females, the highest HR was found in males with PDD (HR 2.3 (95\% CI 1.8-2.8)).

Referrals to liaison psychiatry were associated with reduced survival compared to other mental health services (HR 1.7 (95\% CI 1.5-2.0)).

The AD cohort showed the lowest rates of pneumonia due to aspiration and recurrent falls in males and in females. The highest rate of pneumonia due to aspiration was found in the male PDD group (27\%).

Conclusion. In patients with dementia referred into mental health services, those with $\mathrm{AD}$ survive longer compared to other dementia groups, with PDD patients at highest risk of death. Physical frailty including risk of aspiration, is likely to account for some of this difference in survival.
Treatment of anorexia nervosa in young people with autism: a literature review

\section{Aneesa Karim}

Adolescent Inpatient Unit

doi: 10.1192/bjo.2021.699

Aims. The purpose of this review was to review existing literature relating to treatment of anorexia nervosa in young people with a diagnosis of autism. Hypothesis was that there would be a limited amount of literature in this age group.Previous research has suggested that there is over-representation of autistic traits in anorexia nervosa. There are implications for treatment outcomes for young people with anorexia nervosa and autism. Young people with autism may find it more difficult to engage in psychological treatments for anorexia nervosa, due to cognitive and behavioural inflexibility, or communication difficulties. Researchers are therefore looking at other options for treatment.

Method. This is a narrative review. Search was conducted in January 2020. Keywords used were "anorexia nervosa" combined with "autism" combined with "treatment". Only published, peerreviewed, full articles in English were included. Search of OVID (for MEDLINE, PsycINFO, EMBASE and ERIC databases) gave a result of 222 articles. 9 articles met the inclusion criteria. Search of CINAHL gave a result of 12 articles; 3 articles met the inclusion criteria but had been reviewed following OVID search.

Result. Themes identified for discussion were: cognitive remediation therapy; improving emotional identification; adaptations to communication; dietary, sensory and environmental considerations; recognising the role of autism; and pharmacological therapies.

Conclusion. Literature suggests that treatment targeting cognitive features, common to anorexia nervosa and autism, can be effective. There has been interest in the use of cognitive remediation therapy (CRT) and cognitive remediation and emotion skills training (CREST). However, more research is required in younger patient groups. Use of medication is in experimental stages, with studies considering a role for oxytocin from age 16. Qualitative studies provide information on modifications to treatment which could be helpful. The review highlights the need for a standardised, evidence-based treatment pathway for this patient group.

Attitudes and perceptions of parents towards child and adolescent psychiatric consultation, diagnosis and treatment

Darpan Kaur*, Rishab Verma and Rakesh Ghildiyal

Department of Psychiatry, Mahatma Gandhi Missions Medical College and Hospital, Sector 01, Kamothe, Navi Mumbai, Maharashtra, India

${ }^{*}$ Corresponding author.

doi: 10.1192/bjo.2021.700

Aims. The aim of the study was to assess the attitudes and perceptions of parents towards child and adolescent psychiatric consultation, diagnosis and treatment.

The hypothesis of the study was there are significant problems in the domains of attitude and perceptions of parents towards child and adolescent psychiatric consultation, diagnosis and treatment. Background. Parents are an important stake holder in child and adolescent psychiatry and mental health care service models. There is scarce literature from developing countries regarding 